\title{
Nursing home provision for long-term psychiatric patients
}

\section{Recommendations from the Scottish Division, Royal College of Psychiatrists}

The recommendations were agreed by the Executive Committee of the Scottish Division of the College following detailed advice from its Old Age, Rehabilitation and Mental Handicap Sections. While relating particularly to contracts between Health Boards (authorities) and the independent sector for the continuing care of groups of patients, they may also be relevant to the placement of individuals.

\section{(1) Transfer}

When patients are transferred from hospital the discharging consultant and other members of the multidisciplinary team must be satisfied that the home is adequate for the patient's needs and the move is in the patient's interest. Patients must not be discharged from hospital for administrative or financial reasons. The NHS clinical staff responsible for patients being considered for transfer should be involved in the earliest stages in discussion with any organisation setting up a nursing home.

\section{(2) Location}

The home should be located reasonably near the patient's home and near community services such as shops, transport and recreational facilities. The surroundings should be pleasant in a high amenity area. The grounds should be large enough to allow a degree of exercise without having to venture further into the community. Remote rural locations are generally undesirable and a site on a busy road may be hazardous.

\section{(3) Structure}

(a) The structure must be suitable for sufferers from dementia and other mental illnesses. The disorientation of some residents must be borne in mind.

(b) Single storey accommodation is preferred but otherwise there must be good access to the first floor.

(c) There should be at least one, and preferably several, separate day rooms as well as dining room(s) to allow privacy and small group activities. (d) Sleeping accommodation must allow for privacy, although the need for supervision and the avoiding of unnecessary disturbance to other residents must be borne in mind. Single rooms are highly desirable.

(e) There should be adequate toilet and bathroom facilities allowing for privacy and for mental and physical disabilities.

(f) There should be facilities for visitors.

\section{Staffing}

(a) As the patient's dependency and level of illness are likely to be high, staffing levels must take account of this.

(b) Medical cover. The day-to-day medical cover would be by general practitioners but there would in addition be regular visits by a consultant psychiatrist.

(c) Nursing. There should be an appropriate skill mix of staff with proper training. Shortterm contracts should be avoided and there should not be too many part-time staff. Staffing should be adjusted so that nurses do not have to carry out non-nursing duties.

The level of staff will depend on the degree of disability and behaviour disturbance. Staffing levels should be designed to meet recommended formulae such as the modified Aberdeen or Telford formulae.

(d) There should be access to staff from other disciplines, for example, psychology, occupational therapy, chiropody.

\section{(5) Records}

Proper records should be kept with daily reports. Drug prescriptions must be carefully supervised and recorded with safe facilities for storage.

\section{(6) Quality and inspection}

(a) Quality of care should be maintained. The 'QUEST' document could be used as a quality control.

(b) There should be strong representation on inspection teams from Community Mental 
Health Team members, not only Board officers.

(c) There must be a clear procedure for closing a home where standards are inadequate. The residents must be protected. One option is for the Health Board to take over the care of the residents in the home.

\section{(7) Continuing education}

There should be continuing education and training of all staff in nursing homes.

Approved by Executive and Finance Committee, March 1991

\section{The pharmacist's contribution to the management of substance misuse}

At a recent joint meeting between representatives of the Royal Pharmaceutical Society of Great Britain and the Royal College of Psychiatrists, the contribution of the pharmacist to the management of substance misuse was discussed. It was suggested that each Regional and Local Advisory Committee on substance misuse would benefit from having a community pharmacist member in addition to pharmacists employed by the Health Authorities.

To support this proposal the following points are put forward.

A recent study conducted by Glanz et al (1990) involved a postal survey of a $25 \%$ random sample of community pharmacists in England and Wales. The overall response rate was $79 \%$. The survey indicated that most pharmacists would be prepared to provide equipment to drug injectors and to participate in a local syringe exchange scheme. The survey, having indicated the current involvement of pharmacists, went on to emphasise the potential for building upon that role.

(1) Community pharmacists currently provide dispensing services to most drug addicts

Statistics of Drug Addicts Notified to the Home Office (1989) show that as at December 1988, 12,165 persons were receiving controlled drugs prescribed in treatment for drug addiction.

Of the $25 \%$ random sample of community pharmacies, $23 \%$ of respondents (estimated 2,250 for all pharmacies in England and Wales) were dispensing controlled drugs to persons prescribed these drugs in treatment for drug addiction.

This interaction in itself could provide an opportunity for the provision of information on general healthcare and specific advice on minimising health risks associated with substance misuse.
(2) Community pharmacists can often be the first point of contact for people misusing substances who are not in touch with the substance misuse services

If the local pharmacy is identified as a source of information on substance misuse then the pharmacist's advice might be sought in an informal talk in the first move to tackle a problem by someone reluctant to approach their GP or drug services. The pharmacist is in an ideal position to direct the enquirer to the appropriate expert agency.

(3) Community pharmacists are in a central position to provide advice to the public about substance misuse

Not only advice for the substance misusers but also for anxious parents, spouses, siblings or friends who are concerned about someone close to them whom they believe to be involved in substance misuse.

(4) Community pharmacists are in a central position to provide specific advice to substance misusers about minimising associated health risks

The Glanz survey found that $35 \%$ of pharmacies were asked by at least one drug misuser to sell injecting equipment to them in a four-week period. Thus an estimated 19,647 known or suspected drug misusers would be anticipated to make such a request throughout England and Wales in a four-week period; 676 pharmacists (23\% of respondents) reported that they were currently selling equipment to injecting drug misusers.

(5) Community pharmacists are easily accessible to the public and can provide an appropriate venue for needle exchange schemes

The Monitoring Research Group, Goldsmiths' College Final Report (1988) summarised that syringe exchange schemes have considerable potential to 